\section{How Important is Steady State Cosmology to Classical and Quantum Electrodynamics?}

IN a recent article on cosmology and electrodynamics, Hoyle and Narlikar ${ }^{1}$ have argued that the direct action electrodynamics of Wheeler and Feynman ${ }^{2}$ may be used as evidence that the steady state model of cosmology is the correct form of cosmology in the physical universe (see also ref. 3). They base their argument on the fact that conventional Maxwell-Lorentz electrodynamics, being basically inconsistent because of the presence of selfenergy infinities, can be supplanted only by the WheelerFeynman electrodynamics (the justification of the complete absorber assumption coming automatically out of the steady state model of cosmology). This argument would apparently establish a strong connexion between local electrodynamics and steady state cosmology were it not for the fact that a third and more consistent form of electrodynamics exists (in which the Lorentz-Dirac equation can be derived from a variational principle, without any infinite self-interactions, and without recourse to any cosmological assumptions about complete absorption).

This new formulation of electrodynamics, classical elementary measurement electrodynamics ${ }^{4}$, is based on the paradigm that, in a physical event, it is the mutual measurement interaction between the observer charge and the observed charge which is the basic elementary building block of the theory.

Within the context of a relativistic theory of point charges, this new theory of electrodynamics implies that the charged particles (associated with $\mathbf{J}_{\mu}^{(K)}, K=1,2, \ldots, N$ ) and their electromagnetic fields (associated with $\mathbf{A}_{\mu}{ }^{(K)}$, $K=1,2, \ldots, N)$ are not elementary in themselves but are merely interdependent degrees of freedom in a scalar elementary measurement field $\mathbf{J}_{\mu}^{(K)} \mathbf{A}^{\mu(J)}, K \neq J=1,2, \ldots, N$. Because this implies that the scalar elementary measurement field $\mathbf{J}_{\mu^{(K)}} \mathbf{A}^{\mu(J)}, K \neq J$, is more fundamental than either $\mathbf{J}_{\mu}{ }^{(K)}$ or $\mathbf{A}_{\mu}{ }^{(J)}$ then, in this theory, Maxwell's equations (with the proper Green function) are interpreted to be a set of covariant identities which give a prescription for converting particle currents $\boldsymbol{J}_{\mu}^{(K)}$ into their associated electromagnetic fields $\mathbf{A}_{\mu}^{(I)}$. Because all the electromagnetic fields $\mathbf{A}_{\mu}{ }^{(K)}$ are required to be directly connected to their associated currents, $\mathbf{J}_{\mu}^{(K)}$, through the Maxwell "identities", then no free uncoupled electromagnetic fields will exist in the theory. This means that the phenomena of radiation will occur as the by-product of the propagation of mutual electromagnetic measurement interactions between charged particles and will not be independent of the detector. On the basis of the paradigm which underlies this theory, all self-measurement fields, $\mathbf{J}_{\mu^{(K)}} \mathbf{A}^{\mu(K)} ; K=1,2, \ldots, N$, are excluded $a$ priori as being non-physical. Hence the problem of infinite self-energies is excluded in the basic formulation of the theory and never arises again.

It has been shown ${ }^{4}$ that an action principle for the relativistic elementary measurement of $\mathrm{N}$ classical charged point particles and their associated electromagnetic fields can be constructed. Making the action stationary with respect to the variation of the interacting degrees of freedom gives the equations of motion of the measurement. With the proper choice of Green function for the Maxwell tautologies, the equations of motion of the measurement have the same form as that of WheelerFeynman electrodynamics. They have the distinct advantage, however, that no "complete absorber" assumption is ever needed or used and the electromagnetic fields are not eliminated from the theory a priori as is done in Wheeler-Feymman theory. The difficulties associated with infinite self-energies and mass renormalization are absent from this new theory and a consistent set of energymomentum and angular momentum conservation laws follows from an associated conserved energy-momentum tensor.

This is accomplished in a flat Euclidean universe, in which the effects of radiation reaction can be produced independent of the value of the quantity $\sum_{K=1}^{N}\left(\mathbf{F}_{\mu \nu}{ }^{(K)}(r e t)-\right.$ $\left.\mathbf{F}_{\mu v^{(K)}}(a d v)\right)$. The zero value of this term is usually associated with the complete absorber assumption. Because this theory can reproduce the Lorentz-Dirac equation for point charges, it can reproduce all of the physical predictions of Maxwell-Lorentz theory and Wheeler-Feynman theory, but in a consistent fashion and with fewer assumptions. In addition, following Hoyle and Narlikar, if we quantize only the trajectories of particles and leave the electromagnetic fields as $c$-numbers, this new theory can predict the same results about spontaneous transitions as that of the Hoyle-Narlikar quantum electrodynamics. The details of this have been carried out (my unpublished work). In this work it is shown that the wavemechanical generalization of classical elementary measurement electrodynamics yields a theory which can account for the spontaneous transition process (via its nonlinear structure) even though the associated electromagnetic fields are $c$-numbers and are not second quantized in the usual manner.

The conclusion drawn is that classical elementary measurement electrodynamics (and its quantum general. ization) may exhibit a dependence on cosmology when inserted into a general relativistic framework. It does not, however, require the steady state cosmological model for its internal consistency. Because this new electrodynamic theory offers a superior alternative to Maxwell-Lorentz and Wheeler-Feynman theory, the immediate implication is that Hoyle and Narlikar's argument (that a consistent local electrodynamics does require the steady state cosmology) is not on firm ground. In particular, it remains an open question how important the steady state cosmology is to classical and quantum electrodynamics in the local vicinity.

Department of Physics,

DARRYL J. Leiter

Boston College,

Chestnut Hill, Massachusetts.

Received June 18, 1969.

${ }^{2}$ Hoyle, F., and Narlikar, J. V., Nature, 219, 340 (1968).

${ }^{2}$ Wheeler, J. A., and Feynman, R. P., Rev. Mod. Phys., 17, 157 (1945); ibid., $21,425(1949)$.

${ }^{3}$ Hoyle, F., and Narlikar, J. V., Proc. Roy. Soc., A, 277, 1 (1963).

${ }^{4}$ Leiter, Darryl J., Ann. Phys., 51, 561 (1969).

Hoyle and Narlikar comment as follows:

Maxwell's electromagnetic theory, in spite of its remarkable successes in the experimental field, possesses two main defects. The first is the ad hoc choice of retarded solutions. As long as this choice is made on empirical grounds, we are no better off in our understanding of such basic phenomena as radiation and the electrodynamic arrow of time. The second is the concept of self action which leads to infinities in the study of even the simplest systems. The search for a new theory of electrodynamies should therefore be motivated by two considerations:

1. The new theory must be at least as successful as Maxwell's theory in the experimental field.

2 . The new theory must be free from at least one of the above defects, preferably both.

Our recent work ${ }^{1-3}$ goes some way towards demonstrating that the Wheeler-Feynman theory in a suitable cosmological background fulfils these requirements. In Dr Leiter's elementary measurement electrodynamics the empirical choice of retarded potentials is still retained ${ }^{4}$, and it is therefore no better off than Maxwell's theory in this respect. That he needs no absorber condition is no surprise in the light of this assumption. The absorber condition of Wheeler-Feynman theory has the attractive 